Portland State University

PDXScholar

$9-1-2012$

\title{
Turbulent Concentration Diffusion in Multiphase Flow
}

John D. Ramshaw

Portland State University, jdramshaw@yahoo.com

Follow this and additional works at: https://pdxscholar.library.pdx.edu/phy_fac

Part of the Physics Commons

Let us know how access to this document benefits you.

Citation Details

Ramshaw, J. D. (2012). Turbulent concentration diffusion in multiphase flow. Physics Of Fluids, 24(9), 093301-093301-13.

This Article is brought to you for free and open access. It has been accepted for inclusion in Physics Faculty Publications and Presentations by an authorized administrator of PDXScholar. Please contact us if we can make this document more accessible: pdxscholar@pdx.edu. 


\title{
Turbulent concentration diffusion in multiphase flow
}

\author{
John D. Ramshaw \\ Department of Physics, Portland State University, Portland, Oregon 97207, USA
}

(Received 16 November 2011; accepted 25 July 2012; published online 5 September 2012)

In multifluid multiphase flow models, the velocity of each phase is determined by its own momentum equation, which is coupled to the other phases by pairwise interphase drag forces proportional to velocity differences. When the drag coefficients are large, the phase velocities become nearly equal and the relative motion of the phases becomes diffusional rather than inertial. The multifluid momentum equations then reduce to a single momentum equation for the mixture and a system of linear relations that determine the small residual velocity differences between the phases. We derive such diffusional relations in a very general form that applies to nearly all multiphase flow models of this type. The simplest such models then reduce to "drift flux" models that exhibit pressure diffusion but not ordinary (Fick's law) diffusion driven by concentration gradients. The question then arises of how to consistently account for turbulent concentration diffusion in models of this type. This question is resolved by specializing the general diffusional relations to a rather typical single-pressure multiphase flow model which includes turbulent momentum transport due to Reynolds stresses. This special case serves as a paradigm which illustrates that (a) turbulent concentration diffusion in multiphase flow is a direct consequence of the isotropic (turbulent pressure) part of the Reynolds stresses, provided the latter are expressed in their proper divergence form; and (b) the order of magnitude of the resulting turbulent diffusivities is just what one would anticipate on dimensional grounds. Properly formulated models of this type therefore automatically include turbulent concentration diffusion, and consequently require no further additions or modifications to introduce it. (C) 2012 American Institute of Physics. [http://dx.doi.org/10.1063/1.4748348]

\section{INTRODUCTION}

Multifluid (multifield) models for multiphase flow have found use in a wide variety of diverse scientific and engineering areas, including nuclear reactor safety, mixing of materials due to interfacial instabilities, and many others. In models of this type, the motion of each phase $i$ is described by its own velocity field $\mathbf{u}_{i}$, which is determined by its own momentum equation. Most such models bear a strong family resemblance to one another, but they differ in their details, and those differences are sometimes crucial. However, almost all such models contain interphase drag terms of essentially the same form, which represent momentum exchange between the phases due to pairwise frictional forces. The drag force per unit volume exerted on phase $i$ by phase $j \neq i$ is normally assumed to be of the form

$$
\mathbf{F}_{i j}=-\mathbf{F}_{j i}=\beta_{i j}\left(\left|\mathbf{u}_{j}-\mathbf{u}_{i}\right|\right)\left(\mathbf{u}_{j}-\mathbf{u}_{i}\right) .
$$

The drag coefficients $\beta_{i j}(u)=\beta_{j i}(u)$ are roughly linear functions of $u$ for large $u$ (the gasdynamic drag regime), but are roughly constant for small $u$ (the Stokes drag regime), and approach nonzero limiting values $\beta_{i j}^{0} \equiv \beta_{i j}(0)$ as $u \rightarrow 0$. The total interphase drag force per unit volume on phase $i$ is then simply

$$
\mathbf{F}_{i}=\sum_{j} \mathbf{F}_{i j}=\sum_{j} \beta_{i j}\left(\mathbf{u}_{j}-\mathbf{u}_{i}\right)
$$

in which $j$ ranges from 1 to $N$, where $N$ is the number of phases, and there is no need to exclude the term $j=i$ from the summation since $\mathbf{F}_{i i}=0$. 
The purpose of this paper is to elucidate the simplifications, both generic and specific, that occur when the drag coefficients $\beta_{i j}(u)$ and $\beta_{i j}^{0}$ are sufficiently large that they force the phase velocities $\mathbf{u}_{i}$ to become nearly equal. The multifluid momentum equations then reduce to a single momentum equation for the mixture, together with diffusional expressions that determine the residual relative velocities of the phases. The mathematical structure of the latter expressions depends in an essential way on the assumed form of the drag terms, but it is largely indifferent to the precise forms of the other force terms in the multiphase momentum equations. This circumstance enables us to derive the diffusional relations for the relative phase velocities in a remarkably general form which encompasses essentially all multifluid multiphase flow models containing drag terms of the above form. Those general expressions can then be readily specialized to any particular model of that type.

Multifluid momentum equations of the general form considered here are not inherently restricted to particular multiphase flow regimes or mixture morphologies and are commonly used to describe both separated and dispersed multiphase flows. However, the form of the drag coefficients $\beta_{i j}$ and other constitutive relations depends strongly on the flow regime. As a general rule, the quantities $\beta_{i j}$ are much larger in dispersed flows than separated flows, so they are correspondingly much less likely to be large enough to produce diffusional behavior in separated flows. The practical utility of the present diffusional relations is therefore expected to be primarily limited to dispersed flow, such as bubbles in liquid, droplets in gas, and particulate suspensions.

The diffusional formulation that results when the drag coefficients are sufficiently large is closely analogous, and indeed essentially isomorphic, to the hydrodynamic theory of multicomponent diffusion in gases and plasmas, ${ }^{1,2}$ and the diffusional expressions that we shall derive for the relative phase velocities are simply multiphase analogs of the Stefan-Maxwell equations of molecular diffusion theory. In fact, the present development could readily have been presented in a slightly more general form that would also apply to atomic or molecular mixtures as a special case. However, that additional generality seemed superfluous, since the molecular case has already been presented and discussed in detail. ${ }^{1,2}$

In the multiphase literature, simplified descriptions of the present type are customarily referred to as "drift flux" models or approximations. ${ }^{3-7}$ Such approximations have been widely discussed and employed, but usually in rather restricted and specialized forms which neither reflect nor suggest the generality of the present treatment. Moreover, such approximations are not typically described or regarded as "diffusional," probably because in their simplest and most common forms they do not predict or imply interphase drift velocities proportional to concentration gradients, and hence do not exhibit ordinary (Fick's law) diffusion. This behavior is a direct consequence of the mathematical form of the multiphase pressure gradient terms, which are inherently different from those in molecular mixtures. ${ }^{8,9}$

However, most multiphase flows are turbulent, and one would intuitively expect turbulent velocity fluctuations to produce diffusional mass fluxes of the usual form proportional to concentration gradients. The question then arises of how to consistently describe turbulent concentration diffusion in models of the present type. We address and resolve this question by specializing the general formulation described above to a rather typical single-pressure turbulent multiphase flow model, in which the multiphase momentum equations include force terms due to pressure gradients, interphase drag, virtual mass, Reynolds stresses, and gravitational body forces. It is then straightforward to show that (a) the resulting diffusional expressions for the relative phase velocities contain terms proportional to concentration gradients, which arise from the isotropic part of the Reynolds stresses; and (b) the associated turbulent diffusivities depend on the local turbulent kinetic energy just as one would expect on dimensional grounds.

The detailed analysis of this particular model then serves as a paradigm to illustrate that the essential ingredient required to describe turbulent concentration diffusion in models of this type is simply the isotropic part of the Reynolds stress terms in the multiphase momentum equations, provided that those terms are properly expressed in their natural conservation or divergence form. This conclusion may seem counterintuitive at first, since those terms fundamentally represent the transport of momentum rather than mass, but the present development shows that the former implies the latter in the limit of large friction. 
It must be emphasized, however, that the particular expressions discussed above for describing turbulent concentration diffusion in multiphase mixtures should be regarded as illustrative rather than definitive. Those results are specific to the assumed form of the force terms in the multiphase momentum equations from which they were derived, and they consequently inherit whatever limitations the latter may possess. The particular momentum equations considered here are by no means simplistic, and indeed include reasonable approximations to much of the important physics, but it would hardly be realistic to expect them to be universally valid or satisfactory. The present development should, therefore, not be interpreted as advocating or endorsing the indiscriminate general use of those particular equations or their diffusional simplifications. The essential point is not the form of those particular relations, but rather the fact that the general formulation derived in this paper can readily be specialized to any particular system of multiphase momentum equations containing drag forces of the form of Eq. (2).

The significance and utility of the present formulation depend on whether one intends to directly solve the original multifluid momentum equations in their general form. If so, then one does not of course actually solve the diffusional equations, and the latter simply serve to show the form to which the former reduce when the drag coefficients are large. This knowledge can provide useful insight into the completeness and physical fidelity of the multifluid momentum equations, as the present development illustrates, but the multifluid equations of course remain valid and can still be solved even when $\beta_{i j}$ is large. In that case, however, the equations become stiff and their numerical solution requires the use of implicit time integration. ${ }^{10}$ In contrast, the diffusional relations are only directly useful for practical purposes in the subclass of problems in which all the drag coefficients are uniformly large throughout the entire computational domain, and for all times of interest. In such problems, however, they provide a major simplification of the multifluid description, and significantly reduce the computational effort required to obtain numerical solutions. Moreover, they are more benign and easier to solve, since they are not subject to the notorious instabilities of multifluid models. ${ }^{11}$

The moral of the story is simply this: whatever diffusional behavior multifluid multiphase flow models may or may not possess when the drag coefficients are large, and the form it assumes, are uniquely and automatically implied and determined by the precise form of the multiphase momentum equations and the various force and momentum transfer terms therein. Thus there is no need, and no freedom, to independently postulate diffusional expressions for the relative phase velocities in that limiting case and introduce them into the equations ex post facto. Such expressions will not, in general, be compatible with those implied by the momentum equations, and will thereby destroy the internal consistency of the model. Any physical deficiencies in the description of the diffusional limit must be attributed to corresponding deficiencies in the multiphase momentum equations themselves prior to taking that limit and should be remedied at that level.

\section{THE MULTIPHASE STEFAN-MAXWELL EQUATIONS}

In accordance with the preceding discussion, the multiphase momentum equations that we shall analyze are of the general form

$$
\frac{\partial\left(\alpha_{i} \rho_{i} \mathbf{u}_{i}\right)}{\partial t}+\nabla \cdot\left(\alpha_{i} \rho_{i} \mathbf{u}_{i} \mathbf{u}_{i}\right)=\sum_{j} \beta_{i j}\left(\mathbf{u}_{j}-\mathbf{u}_{i}\right)+\mathbf{T}_{i},
$$

where $\alpha_{i}$ and $\rho_{i}$ are, respectively, the volume fraction and intrinsic mass density of phase $i$, and $\mathbf{T}_{i}$ is the local time rate of change of the momentum of phase $i$ per unit volume due to all forces and momentum transfer mechanisms other than convection and interphase drag forces. Thus $\mathbf{T}_{i}$ includes pressure gradients, body forces, and in general various other model-specific terms; e.g., virtual mass forces and Reynolds stresses. We shall also require the multiphase continuity equations, and for simplicity we restrict attention to situations in which there is no mass exchange between the phases. The continuity equations then assume the familiar form

$$
\frac{\partial\left(\alpha_{i} \rho_{i}\right)}{\partial t}+\nabla \cdot\left(\alpha_{i} \rho_{i} \mathbf{u}_{i}\right)=0
$$


Summing Eq. (4) over all phases $i$, we obtain the mixture continuity equation in the usual form

$$
\frac{\partial \rho}{\partial t}+\nabla \cdot(\rho \mathbf{u})=0
$$

where $\rho=\sum_{i} \alpha_{i} \rho_{i}$ is the total mass density, $\rho \mathbf{u}=\sum_{i} \alpha_{i} \rho_{i} \mathbf{u}_{i}$, and $\mathbf{u}$ is the mass-weighted velocity of the mixture, which also has the significance of the local value of the mixture momentum per unit mass.

In single-pressure multiphase flow models, to which our attention here is restricted, Eqs. (3) and (4) constitute $2 N$ equations in the $3 N$ primary independent variables $\mathbf{u}_{i}, \alpha_{i}$ (only $N-1$ of which are independent, since they sum to unity), $\rho_{i}$, and the pressure $p$ (the gradient of which appears in $\mathbf{T}_{i}$ ). When the phases are incompressible, the additional $N$ equations required to obtain a closed equation system are provided by the known or given values of the phase densities $\rho_{i}$. In compressible multiphase flow, the $\rho_{i}$ are no longer known and the specific internal energies $e_{i}$ of the phases appear as $N$ additional independent variables, so that $2 N$ additional equations are required to obtain a closed system. Those $2 N$ equations are provided by $N$ energy evolution equations and $N$ thermodynamic state relations of the form $p=f_{i}\left(\rho_{i}, e_{i}\right)$, where the state functions $f_{i}$ are presumed to be known. The drag coefficients $\beta_{i j}$ appearing in Eqs. (1) and (2) are presumed to be known or given functions of $\left|\mathbf{u}_{j}-\mathbf{u}_{i}\right|$ and the other dependent variables. Any further variables appearing in $\mathbf{T}_{i}$ (including turbulence variables) are presumed to be either known or determined by corresponding further evolution equations (such as a turbulence model), but the precise form of those equations need not be specified for present purposes.

The multiphase flows of present interest are turbulent, so the dependent variables in Eqs. (1)- (5) and subsequent equations should be interpreted as appropriately weighted ensemble averages over the turbulent fluctuations. Unweighted ensemble averages will be denoted by angular brackets; viz. $\langle\cdots\rangle$. Just as in single-phase turbulence, ${ }^{12}$ the equations assume a much simpler form if mass-weighted or Favre averaging is used, ${ }^{12,13}$ and this will be understood in what follows. Thus the ensemble-averaged values of the variables $\alpha_{i}, \rho_{i}$, and $\mathbf{u}_{i}$ are defined by $\left\langle\alpha_{i}\right\rangle,\left\langle\alpha_{i} \rho_{i}\right\rangle /\left\langle\alpha_{i}\right\rangle$, and $\left\langle\alpha_{i} \rho_{i} \mathbf{u}_{i}\right\rangle /\left\langle\alpha_{i} \rho_{i}\right\rangle$, respectively. (Note that we do not use separate symbols to distinguish between averaged and unaveraged variables. It will simply be understood that all variables are ensemble averages unless they appear inside angular brackets, where they denote the corresponding unaveraged local instantaneous variables.) As a result of the above definitions, the ensemble-averaged continuity equations (4) and (5) are identical in form to the corresponding unaveraged equations, and consequently contain no Reynolds-like mass flux terms analogous to the Reynolds stress terms in the ensemble-averaged momentum equations. Thus there is no mass flux or turbulent diffusion of phase $i$ relative to its own mean velocity $\mathbf{u}_{\mathbf{i}}$. The turbulent diffusion of present interest refers to the motion of phase $i$ relative to the mass-weighted mean velocity $\mathbf{u}$ of the multiphase mixture. The corresponding mass flux is simply $\mathbf{J}_{i} \equiv \alpha_{i} \rho_{i}\left(\mathbf{u}_{i}\right.$ $-\mathbf{u})$, which becomes diffusional in character when the drag coefficients $\beta_{i j}$ are sufficiently large. All these features are closely analogous to the hydrodynamic theory of atomic/molecular diffusion in multicomponent gas mixtures. ${ }^{1,2}$

The first step in the derivation is to convert Eq. (3) into so-called nonconservative form by combining it with Eq. (4) to obtain

$$
\alpha_{i} \rho_{i} \frac{D_{i} \mathbf{u}_{i}}{D t}=\sum_{j} \beta_{i j}\left(\mathbf{u}_{j}-\mathbf{u}_{i}\right)+\mathbf{T}_{i}
$$

where $D_{i} / D t \equiv \partial / \partial t+\mathbf{u}_{i} \cdot \nabla$ is the convective time derivative following phase $i$. The quantity $D_{i} \mathbf{u}_{i} / D t$ is simply the local Lagrangian acceleration of phase $i$.

It is clear by inspection of Eq. (6) that when the drag coefficients $\beta_{i j}$ are large, the velocity differences $\mathbf{u}_{j}-\mathbf{u}_{i}$ must correspondingly become small. Of course, in order for the $\beta_{i j}$ to remain large enough to maintain those small velocity differences, their limiting values $\beta_{i j}^{0} \equiv \beta_{i j}(0)$ must likewise be large in the same sense. The phase velocities $\mathbf{u}_{i}$ become equal in the limit as $\beta_{i j}^{0} \rightarrow \infty$, so that $\mathbf{u}_{i}=\mathbf{u}$ in that limit. Diffusional or drift flux approximations are asymptotic quasi-steady approximations obtained by replacing the $\mathbf{u}_{i}$ by their common limiting value $\mathbf{u}$ in the Lagrangian 
accelerations $D_{i} \mathbf{u}_{i} / D t$, but nowhere else. When this is done, Eq. (6) becomes

$$
\alpha_{i} \rho_{i} \frac{D \mathbf{u}}{D t}=\sum_{j} \beta_{i j}\left(\mathbf{u}_{j}-\mathbf{u}_{i}\right)+\mathbf{T}_{i},
$$

where $D / D t=\partial / \partial t+\mathbf{u} \cdot \nabla$ is the usual convective time derivative following the mass-weighted mean velocity of the mixture. In accordance with the preceding discussion, it is understood that if $\mathbf{T}_{i}$ depends on the accelerations $D_{i} \mathbf{u}_{i} / D t$, as it typically does in the case of virtual mass terms, ${ }^{13-15}$ then those accelerations are also to be replaced by $D \mathbf{u} / D t$ therein. Summing Eq. (7) over all phases $i$, we obtain the total momentum equation for the mixture,

$$
\rho \frac{D \mathbf{u}}{D t}=\sum_{i} \mathbf{T}_{i}
$$

which determines u. Combining Eqs. (7) and (8), we obtain

$$
\sum_{j} \beta_{i j}\left(\mathbf{u}_{j}-\mathbf{u}_{i}\right)=-\left(\mathbf{T}_{i}-y_{i} \sum_{j} \mathbf{T}_{j}\right),
$$

where $y_{i} \equiv \alpha_{i} \rho_{i} / \rho$ is the local mass fraction of phase $i$. Equations (9) are the multiphase StefanMaxwell equations in their most general form. Only $N-1$ of them are independent, since their summation over $i$ yields $0=0$. The remaining equation required to obtain a closed system for the phase velocities $\mathbf{u}_{i}$ is the relation $\sum_{i} y_{i} \mathbf{u}_{i}=\mathbf{u}$.

The possible dependence of $\mathbf{T}_{i}$ on $D \mathbf{u} / D t$, as discussed above, introduces a potential complication which has no analog in atomic and molecular mixtures. If such is the case, then Eq. (8) does not explicitly determine $D \mathbf{u} / D t$, but rather constitutes an implicit equation for $D \mathbf{u} / D t$, which must first be solved in order to determine $\mathbf{u}$ and to evaluate the quantities $\mathbf{T}_{i}$ in Eq. (9). Fortunately, however, this unwelcome complication rarely if ever occurs in practice, because the only common terms in $\mathbf{T}_{i}$ that depend on the phase accelerations $D_{i} \mathbf{u}_{i} / D t$ are virtual mass terms, which are almost invariably assumed to depend only on differences between those accelerations. ${ }^{13-15}$ Those differences vanish when $D_{i} \mathbf{u}_{i} / D t=D \mathbf{u} / D t$, so that neither they nor $\mathbf{T}_{i}$ itself depend on the actual value of $D \mathbf{u} / D t$, which moreover is then explicitly given by Eq. (8).

Since the velocity differences $\mathbf{u}_{j}-\mathbf{u}_{i}$ are small, Eq. (9) may be simplified by linearizing it in those small differences, with the result

$$
\sum_{j} \beta_{i j}^{0}\left(\mathbf{u}_{j}-\mathbf{u}_{i}\right)=-\left(\mathbf{T}_{i}-y_{i} \sum_{j} \mathbf{T}_{j}\right),
$$

where it will henceforth be understood that the quantities $\mathbf{T}_{i}$ have been linearized in $\mathbf{u}_{j}-\mathbf{u}_{i}$ or $\mathbf{u}_{i}$ $-\mathbf{u}$. However, $\mathbf{T}_{i}$ is often either independent of or linear in the $\mathbf{u}_{i}$, in which case it is unchanged by linearization. Equations (10), together with the relation $\mathbf{u}=\sum_{i} y_{i} \mathbf{u}_{i}$, constitute a closed system of linear algebraic equations that determine the velocities $\mathbf{u}_{i}$.

It is clear from Eq. (10) that if $\mathbf{T}_{i}=0$ for all $i$, then the phase velocities $\mathbf{u}_{i}$ are all equal, so that $\mathbf{u}_{i}=\mathbf{u}$ for all $i$. The quantities $\mathbf{T}_{i}$ therefore play the role of driving forces for the relative motion of the phases. Moreover, Eq. (10) is linear in both $\mathbf{u}_{i}$ and $\mathbf{T}_{i}$, so the velocity differences $\mathbf{u}_{i}-\mathbf{u}_{j}$ are likewise linear in the driving forces $\mathbf{T}_{i}$. The precise mathematical form of the relative velocities is entirely and uniquely determined by the mathematical form of the $\mathbf{T}_{i}$, so it is apparent that only terms appearing in the $\mathbf{T}_{i}$ can appear in the relative velocities (but with different coefficients, of course). It is customary to describe the relative motion of the phases as "diffusional," in the usual Fick's law sense, when the relative velocities contain terms proportional to concentration gradients. (Similarly, thermal, pressure, and forced diffusion refer to the presence of terms proportional to temperature and pressure gradients and external forces, respectively.) In the present context, the primary concentration variables are the volume fractions $\alpha_{i}$. It follows that in order for ordinary concentration diffusion to occur, $\mathbf{T}_{i}$ must contain terms proportional to $\nabla \alpha_{i}$. This is not the case in the very simplest single-pressure multiphase flow models, in which $\mathbf{T}_{i}=-\alpha_{i} \nabla p$. (Such models are actually ill-posed, but that is an unrelated issue which was resolved long ago. ${ }^{11}$ ) Since $\mathbf{T}_{i}$ does not 
contain terms proportional to $\nabla \alpha_{i}$, such models do not exhibit ordinary concentration diffusion, but merely pressure diffusion. In contrast, the corresponding term in atomic and molecular mixtures is $-\nabla\left(\alpha_{i} p\right)=-\alpha_{i} \nabla p-p \nabla \alpha_{i},{ }^{8,9,16}$ which gives rise to both concentration and pressure diffusion. ${ }^{1,2}$ If one believes on physical grounds that concentration diffusion should occur in multiphase flow (e.g., when the flow is turbulent), the proper way to introduce and represent it in the model is to seek physically motivated terms proportional to $\nabla \alpha_{i}$ which should in principle appear in $\mathbf{T}_{i}$ but were previously neglected. These general considerations will be illustrated in detail in Sec. III.

\section{TURBULENT CONCENTRATION DIFFUSION}

We now proceed to specialize the general formulation derived in Sec. II to a rather typical single-pressure model for turbulent multiphase flow, in which $\mathbf{T}_{i}$ includes terms representing forces and momentum fluxes due to pressure gradients, virtual mass, Reynolds stresses, and gravity. This specialization provides a detailed illustration of how the general formalism may be applied to particular special cases, and the specific model that we shall consider will serve as a paradigm to illustrate that the essential ingredient required to describe turbulent concentration diffusion in models of this type is simply the isotropic part of the Reynolds stress terms in the multiphase momentum equations.

The particular model that we shall analyze is defined by

$$
\mathbf{T}_{i}=-\alpha_{i} \nabla p+\mathbf{V}_{i}+\nabla \cdot \mathbf{R}_{i}+\alpha_{i} \rho_{i} \mathbf{g},
$$

where $\mathbf{V}_{i}$ is the force per unit volume exerted on phase $i$ by the other phases $j$ due to virtual mass effects, $\mathrm{R}_{i}$ is the Reynolds stress tensor for phase $i$, and $\mathbf{g}$ is the external gravitational body force per unit mass. Equation (11) obviously does not include all forces and momentum transfer mechanisms known to occur in multiphase flows; e.g., particle stresses in suspensions. ${ }^{17,18}$ Such additional terms and effects lie outside the scope of this paper, but we re-emphasize that the general formulation developed in Sec. II provides a framework whereby the diffusional effects produced by any given or assumed form of $\mathbf{T}_{i}$ can be systematically derived.

As discussed in Sec. II, we shall suppose that $\mathbf{V}_{i}$ depends only on differences between the Lagrangian accelerations of the phases, and consequently makes no contribution to Eqs. (7)-(10) and may henceforth be neglected. Equation (11) then reduces to

$$
\mathbf{T}_{i}=-\alpha_{i} \nabla p+\nabla \cdot \mathbf{R}_{i}+\alpha_{i} \rho_{i} \mathbf{g} .
$$

To proceed further, we require a suitable expression for $\mathrm{R}_{i}$, which arises in the usual way from the ensemble average of the convective momentum flux in Eq. (3), and is formally given by

$$
\mathrm{R}_{i}=-\left\langle\alpha_{i} \rho_{i} \mathbf{u}_{i}^{\prime} \mathbf{u}_{i}^{\prime}\right\rangle,
$$

where $\mathbf{u}_{i}^{\prime}$ is the deviation of the local instantaneous value of $\mathbf{u}_{i}$ from its mass-weighted (Favre averaged) mean value due to turbulent fluctuations, ${ }^{12}$ and $\langle\cdots\rangle$ denotes an appropriate ensemble average. It is convenient to decompose $\mathbf{R}_{i}$ into isotropic and traceless parts:

$$
\mathrm{R}_{i}=-q_{i} \mathrm{U}+\boldsymbol{\Omega}_{i},
$$

where $U$ is the unit dyadic, whose Cartesian components $U_{\mu \nu}$ are unity if $\mu=v$ and zero otherwise, and the deviatoric Reynolds stresses $\boldsymbol{\Omega}_{i}$ are required to satisfy $\boldsymbol{\Omega}_{i}: \mathrm{U}=\operatorname{Tr}\left(\boldsymbol{\Omega}_{i}\right)=0$, where $\operatorname{Tr}(\mathrm{M})=\sum_{\mu} M_{\mu \mu}$. It follows that

$$
-3 q_{i}=\mathrm{R}_{i}: \mathrm{U}=-\left\langle\alpha_{i} \rho_{i}\left|\mathbf{u}_{i}^{\prime}\right|^{2}\right\rangle .
$$

But $\frac{1}{2}\left\langle\alpha_{i} \rho_{i}\left|\mathbf{u}_{i}^{\prime}\right|^{2}\right\rangle$ is simply the mean turbulent kinetic energy per unit volume of phase $i$, which may also be written as $\alpha_{i} \rho_{i} k_{i}$, where $k_{i}$ is the mass-weighted mean kinetic energy per unit mass of phase $i$, and $\alpha_{i}$ and $\rho_{i}$ denote their ensemble-averaged mean values $\left\langle\alpha_{i}\right\rangle$ and $\left\langle\alpha_{i} \rho_{i}\right\rangle /\left\langle\alpha_{i}\right\rangle$, respectively, as discussed in Sec. II. Thus

$$
q_{i}=\frac{2}{3} \alpha_{i} \rho_{i} k_{i}
$$


Inspection of Eqs. (6), (11), and (14) shows that the isotropic part of $\mathbf{R}_{i}$ introduces a term $-\nabla q_{i}$ into the momentum equation for phase $i$, so that $q_{i}$ plays the role of a turbulent partial pressure of phase $i$. This in turn introduces a term $-\nabla q$ into the total momentum equation (8), where $q=\sum_{i} q_{i}$, so that $q$ plays the role of the turbulent pressure of the mixture. The total turbulent kinetic energy per unit volume is $\rho k \equiv \sum_{i} \alpha_{i} \rho_{i} k_{i}$, so that $k=\sum_{i} y_{i} k_{i}$ is the turbulent kinetic energy per unit mass of the mixture. These relations combine to imply that $q=\frac{2}{3} \rho k$.

It is cumbersome to separately model and compute the $k_{i}$ for each phase individually, so this is seldom done, but most modern turbulence models do compute $k$. In such situations, it becomes necessary to approximate the $k_{i}$ in terms of $k$. The simplest physically reasonable approximation of this type appears to be that $\rho_{i} k_{i}$ has the same value for all phases, independently of $i$. It then follows that $\rho_{i} k_{i}=\rho k$ for all $i$. This approximation states that the turbulent kinetic energy of phase $i$ per unit volume occupied by phase $i$ is the same for all phases, which can be motivated and made plausible in at least two ways. First, it implies that the mean-square turbulent velocity fluctuations within each phase are inversely proportional to the mass density thereof, which is reminiscent of the equipartition of energy in statistical mechanics and the kinetic theory of gases. This is intuitively and aesthetically appealing, but lacks a sound theoretical basis in the present context. Second, it implies that the effective turbulent pressures within each phase have equilibrated. In much the same way as $q_{i}=\frac{2}{3} \alpha_{i} \rho_{i} k_{i}$ is the turbulent partial pressure of phase $i$ as discussed above, $\frac{2}{3} \rho_{i} k_{i}$ plays the role of the effective turbulent pressure within phase $i$ (just as it does in single-phase turbulence ${ }^{12}$ ), and if the latter pressures are equal, then so are the quantities $\rho_{i} k_{i}$. We therefore adopt the approximation $\rho_{i} k_{i}=\rho k$, whereupon Eq. (16) reduces to $q_{i}=\frac{2}{3} \alpha_{i} \rho k=\alpha_{i} q$.

Equation (14) shows that both $q_{i}$ and $\boldsymbol{\Omega}_{i}$ contribute to $\mathbf{R}_{i}$, and thereby to $\mathbf{T}_{i}$ via Eq. (12) and the relative velocities of the phases via Eq. (10). In general, both of those contributions are quantitatively significant, but we shall show below that a nonzero $q_{i}$ or $k_{i}$ is the sole essential ingredient required to produce turbulent concentration diffusion, whereas $\boldsymbol{\Omega}_{i}$ may be regarded as a correction term in that sense, albeit not necessarily a small one. To confirm these assertions, we shall proceed to neglect $\boldsymbol{\Omega}_{i}$ for simplicity. The additional terms to which $\boldsymbol{\Omega}_{i}$ gives rise are worked out in the Appendix. When $\boldsymbol{\Omega}_{i}$ is neglected, Eq. (14) reduces to $\mathrm{R}_{i}=-q_{i} \mathrm{U}$, which combines with Eq. (12) to yield

$$
\begin{aligned}
\mathbf{T}_{i} & =-\alpha_{i} \nabla p-\nabla q_{i}+\alpha_{i} \rho_{i} \mathbf{g} \\
& =-\alpha_{i} \nabla p-\nabla\left(\alpha_{i} q\right)+\alpha_{i} \rho_{i} \mathbf{g} .
\end{aligned}
$$

The multiphase Stefan-Maxwell equations for this particular model are now obtained simply by combining Eq. (17) with Eq. (10). We thereby obtain, after a little algebra,

$$
\sum_{j} \beta_{i j}^{0}\left(\mathbf{u}_{j}-\mathbf{u}_{i}\right)=\left(\alpha_{i}-y_{i}\right) \nabla(p+q)+q \nabla \alpha_{i} .
$$

In accordance with the preceding discussion, we see that the relative phase velocities contain terms proportional to $\nabla(p+q)$ and $\nabla \alpha_{i}$. The former terms represent pressure diffusion (based on the sum of the thermodynamic and turbulent pressures, just as one would intuitively expect), while the latter represent concentration diffusion, which is seen to arise as a direct consequence of the isotropic part of the Reynolds stress, and which would vanish if $k$ were zero. Note that in this model, the driving force for concentration diffusion is $\nabla \alpha_{i}$ rather than either $\nabla y_{i}$ or $\nabla\left(\alpha_{i} \rho_{i}\right)$. Note also that terms involving $\mathbf{g}$ have canceled out, so that there is no forced diffusion due to gravity. (This feature is not peculiar to the present model, but is quite general.) However, gravity influences the pressure gradient, so it indirectly affects the phase velocities and mass fluxes in this manner. In the special case of hydrostatic equilibrium, $\nabla p=\rho \mathbf{g}$, so that $\nabla p$ can be eliminated in favor of $\mathbf{g}$, but this is not possible in general. It follows that drift flux approximations for the diffusion fluxes that directly involve $\mathbf{g}$ are not fundamentally based, and implicitly assume hydrostatic equilibrium, or at least a hydrostatic pressure field.

It is convenient to manipulate Eq. (18) into a form which more closely resembles the StefanMaxwell equations for atomic and molecular mixtures, in which it is customary to introduce binary diffusion coefficients $D_{i j}$ in lieu of the drag coefficients $\beta_{i j}^{0}{ }^{1,2}$ Equation (18) is already very similar in form to the corresponding equation in atomic and molecular mixtures, ${ }^{1,2}$ and a comparison between 
the two suggests that the appropriate definition of $D_{i j}$ in the present context is

$$
D_{i j}=\frac{q \alpha_{i} \alpha_{j}}{\beta_{i j}^{0}}
$$

Note that $D_{i j}$ has the usual dimensions of diffusivity, namely, $\mathrm{m}^{2} / \mathrm{s}$. Moreover, it is of precisely the expected order of magnitude for a turbulent diffusivity, which one would expect on dimensional grounds to be of order $k \tau$, where $\tau$ is an appropriate characteristic time scale. Inspection of Eq. (6) shows that the relaxation time scale for equilibration of the phase velocities is of order $\tau=\rho / \beta$, where $\beta$ is a typical drag coefficient. Thus $k \tau \sim(q / \rho)(\rho / \beta)=q / \beta$, which is just the order of magnitude of the diffusivities defined by Eq. (19). Eliminating $\beta_{i j}^{0}$ from Eq. (18) in favor of $D_{i j}$, we obtain

$$
\sum_{j} \frac{\alpha_{i} \alpha_{j}}{D_{i j}}\left(\mathbf{u}_{j}-\mathbf{u}_{i}\right)=\left(\alpha_{i}-y_{i}\right) q^{-1} \nabla(p+q)+\nabla \alpha_{i} \equiv \mathbf{G}_{i} .
$$

Of course, Eq. (20) should only be used in situations where $q$ and hence $k$ are always nonzero, whereas Eq. (18) is more fundamental and remains applicable even in the special case $k=0$.

In order to verify that $D_{i j}$ has the significance of a concentration diffusion coefficient, it is instructive to consider the special case in which all the binary diffusivities $D_{i j}$ are equal with the common value $D$. The resulting expressions also apply to the different special case of binary (two-phase) mixtures, since there is only a single binary diffusivity $D_{12}=D_{21}=D$ when $N=2$. Equation (20) then becomes

$$
\sum_{j} \alpha_{i} \alpha_{j}\left(\mathbf{u}_{j}-\mathbf{u}_{i}\right)=D \mathbf{G}_{i}
$$

which readily simplifies to

$$
\alpha_{i}\left(\mathbf{u}_{i}-\mathbf{u}_{v}\right)=-D \mathbf{G}_{i},
$$

where $\mathbf{u}_{v} \equiv \sum_{i} \alpha_{i} \mathbf{u}_{i}$ is the volume-weighted velocity of the mixture. The mass flux of phase $i$ relative to $\mathbf{u}_{v}$ is given by

$$
\mathbf{J}_{i}^{v} \equiv \alpha_{i} \rho_{i}\left(\mathbf{u}_{i}-\mathbf{u}_{v}\right)=-\rho_{i} D \mathbf{G}_{i}
$$

in terms of which the continuity equation for phase $i$, Eq. (4), may be rewritten in the equivalent form

$$
\frac{\partial\left(\alpha_{i} \rho_{i}\right)}{\partial t}+\nabla \cdot\left(\alpha_{i} \rho_{i} \mathbf{u}_{v}\right)=-\nabla \cdot \mathbf{J}_{i}^{v}=\nabla \cdot\left(\rho_{i} D \mathbf{G}_{i}\right)
$$

The value of $\mathbf{u}_{v}$ is not known a priori and is not determined by Eq. (22) or (23), but it may readily be computed from $\mathbf{u}$ by means of the easily verified identity

$$
\rho \mathbf{u}_{v}=\rho \mathbf{u}-\sum_{i} \mathbf{J}_{i}^{v} .
$$

In the incompressible case in which $\rho_{i}$ is a constant, Eq. (24) further reduces to

$$
\frac{\partial \alpha_{i}}{\partial t}+\nabla \cdot\left(\alpha_{i} \mathbf{u}_{v}\right)=\nabla \cdot\left(D \mathbf{G}_{i}\right)
$$

In the absence of pressure diffusion (i.e., when $\alpha_{i}=y_{i}$ or $\left.\nabla(p+q)=0\right), \mathbf{G}_{i}=\nabla \alpha_{i}$ and Eq. (26) simplifies to

$$
\frac{\partial \alpha_{i}}{\partial t}+\nabla \cdot\left(\alpha_{i} \mathbf{u}_{v}\right)=\nabla \cdot\left(D \nabla \alpha_{i}\right)
$$

which is simply a standard convective diffusion equation for $\alpha_{i}$.

Unfortunately, when the diffusivities $D_{i j}$ are unequal, as they normally are, it is necessary to solve the full coupled linear system of Eqs. (20), together with the relation $\sum_{i} y_{i} \mathbf{u}_{i}=\mathbf{u}$, to obtain the phase velocities $\mathbf{u}_{i}$ and thereby the mass fluxes $\mathbf{J}_{i}^{v}$ and the volume-weighted velocity $\mathbf{u}_{v}$. In bygone days, the self-consistent effective binary diffusion approximation ${ }^{19,20}$ was commonly used to avoid 
solving such systems. Nowadays, however, the easy availability of free linear algebra software such as LAPACK ${ }^{21}$ has greatly reduced the motivation for using such approximations.

\section{COMPARISONS WITH PREVIOUS WORK}

In this section, we compare and contrast the present results with the relevant earlier work of which we are aware. The discussion is limited to work in which the large-friction or diffusional limit of multifluid multiphase flow models is specifically addressed.

\section{A. The Besnard-Harlow model}

Besnard and Harlow $(\mathrm{BH})^{22}$ described a turbulence model for incompressible two-phase flow, which in spite of the latter restrictions is far more elaborate than the model of Sec. III. However, the BH model simplifies to a tractable form in the limit of large friction (which they refer to as the strong coupling limit), in which they deduce that the relative motion of the phases becomes diffusional in the ordinary Fick's law sense, and that the turbulent diffusivity is proportional to the Reynolds stresses and thereby to the turbulent kinetic energy, in qualitative agreement with the results of Sec. III. The resulting convective diffusion equation for the volume fractions is given by their Eq. (67). In order to quantitatively compare this result with our corresponding Eq. (26), we must first discard the anisotropic parts of the Reynolds stresses, just as we did in Sec. III. When this is done, their Eq. (67) takes the form, in our notation,

$$
\frac{\partial \alpha_{i}}{\partial t}+\nabla \cdot\left(\alpha_{i} \mathbf{u}_{v}\right)=\rho_{i} \rho_{j} \nabla \cdot\left(\rho^{-1} D \nabla \alpha_{i}\right)
$$

where $j=3-i$. This differs from our Eq. (26) in two respects: (a) the pressure diffusion term is missing, and (b) the concentration diffusion term is different in form, and moreover is dimensionally incorrect, which suggests the possibility of a typographical or algebraic error in their Eq. (67). We therefore endeavored to retrace the steps of their derivation on p. 694, and we thereby obtained a different result, namely,

$$
\frac{\partial \alpha_{i}}{\partial t}+\nabla \cdot\left(\alpha_{i} \mathbf{u}_{v}\right)=\nabla \cdot\left[q^{-1} D \nabla\left(q y_{i}\right)\right]
$$

However, we found no errors in the equations leading up to their Eq. (67), so the discrepancy between Eqs. (28) and (29) seems to have been introduced during the omitted steps between their Eqs. (66) and (67). The concentration diffusion term in Eq. (29) is now dimensionally correct, but it still differs in form from that in our Eq. (26), and the pressure diffusion term remains absent without leave. Upon further scrutiny, those discrepancies were found to be the net result of two interacting factors, one of which is incidental, while the other is essential and of critical importance. The incidental factor is that their Eqs. (62) and (63) combine to imply, in our notation, that $k_{1}=$ $k_{2}=k$, whereas our Eq. (26) is based on the alternative relation $\rho_{1} k_{1}=\rho_{2} k_{2}=\rho k$. We prefer the latter relation for the reasons discussed in Sec. III, but the former is not obviously untenable. However, the essential factor responsible for the discrepancies between Eqs. (26) and (29) is the mathematical procedure by which $\mathrm{BH}$ imposed the strong coupling limit, which resulted in the inadvertent loss of pressure diffusion and damaged the concentration diffusion fluxes as well. This procedure was simply a formal expansion in powers of $\beta_{12}^{-1}$, but it was based on the unaccountable presumption that the Reynolds stresses, like the drag terms, are of order $\beta_{12}$ while all other terms in the momentum equations are of order unity. BH offer no physical rationale for that presumption, and indeed no such rationale is apparent. Mathematically, however, this has the effect of forcing the Reynolds stress terms to balance the drag terms in the limit $\beta_{12} \rightarrow \infty$. This is precisely the content of their Eqs. (60) and (61), and in our notation is equivalent (for $N=2$ ) to the relation

$$
\beta_{i j}\left(\mathbf{u}_{j}-\mathbf{u}_{i}\right)=-\nabla \cdot \mathbf{R}_{i} .
$$

This discards more of the terms in the Stefan-Maxwell equations than it retains, including the summation in the right member of Eq. (9), which ensures consistency with the total momentum 
equation (8). As a result, Eq. (30) is not even self-consistent unless $\nabla \cdot\left(R_{1}+R_{2}\right)=0$, and imposing that condition would remove the Reynolds stress terms from the total momentum equation, which is clearly unacceptable.

\section{B. The Youngs model}

In a series of papers, ${ }^{23-25}$ Youngs has described an evolving multiphase flow model in which the momentum equations contain essentially the same basic physical ingredients as the model discussed in Sec. III, and which also describes turbulent concentration diffusion, as well as pressure diffusion, in the limit of large friction. However, the two models nevertheless differ significantly in the following important details, and consequently predict entirely different expressions for the velocities and diffusional mass fluxes of the phases.

\section{The Reynolds stress terms}

In our notation, Youngs takes the Reynolds stress term in $\mathbf{T}_{i}$ to be of the form $y_{i} \nabla \cdot \mathbf{R}$, where $\mathrm{R}=\sum_{i} \mathbf{R}_{i}$ is the total Reynolds stress tensor for the mixture. ${ }^{24,25}$ The corresponding term in Eq. (11) is $\nabla \cdot R_{i}$. Both forms sum to $\nabla \cdot R$ in Eq. (8), but otherwise disagree. It is easy to see that the term $y_{i} \nabla \cdot \mathbf{R}$ drops out of Eq. (9) and consequently produces no relative motion of the phases and no turbulent concentration diffusion. In contrast, the development of Sec. III shows that the isotropic part of $\nabla \cdot \mathbf{R}_{i}$ is in fact the basic driving force for concentration diffusion. However, this feature of the Youngs model was not an unintentional oversight; the form $y_{i} \nabla \cdot \mathbf{R}$ was deliberately designed to cancel out and make no contribution to the relative phase velocities in the limit of large friction. ${ }^{24,25}$ At the same time, Youngs manually introduced concentration diffusion into the model in a different way by modifying the form of the drag terms, as discussed below. The latter modification would have been entirely unnecessary if the Reynolds stress terms (or merely their isotropic parts) had been included in the multiphase momentum equations in their proper conservative or divergence form $\nabla \cdot \mathrm{R}_{i}$. As discussed in Sec. III, the latter form is an immediate consequence of ensemble averaging the momentum convection term in Eq. (3) and cannot be mathematically transformed into the form assumed by Youngs.

\section{The drag forces}

The initial version of the Youngs mode ${ }^{23}$ contained interphase frictional forces of the standard form given in Eq. (1). That early version of the model did not include Reynolds stresses or other force terms proportional to concentration gradients, and consequently did not exhibit concentration diffusion, as discussed in Sec. II. In order to introduce turbulent concentration diffusion into the model, Youngs subsequently ${ }^{24,25}$ modified the form of the drag forces by replacing $\mathbf{u}_{i}$ therein with $\mathbf{u}_{i}-\mathbf{w}_{i}$, where $\mathbf{w}_{i}$ is a diffusional velocity of the postulated form

$$
\mathbf{w}_{i}=-\frac{D}{\alpha_{i} \rho_{i}} \nabla\left(\alpha_{i} \rho_{i}\right)
$$

and $D$ is a turbulent diffusivity. This modification has the effect that when the drag coefficients become infinitely large, the differences between the phase velocities no longer vanish but rather approach the finite limiting values

$$
\mathbf{u}_{i}-\mathbf{u}_{j}=\mathbf{w}_{i}-\mathbf{w}_{j}
$$

from which it readily follows that

$$
\mathbf{u}_{i}-\mathbf{u}_{v}=\mathbf{w}_{i}-\sum_{j} \alpha_{j} \mathbf{w}_{j} .
$$

Youngs originally interpreted $\mathbf{w}_{i}$ as $\mathbf{u}_{i}-\mathbf{u}_{v},{ }^{24}$ but that interpretation is untenable because it would require $\mathbf{w}_{i}$ to satisfy the constraint $\sum_{i} \alpha_{i} \mathbf{w}_{i}=0$, which is violated by Eq. (31) (except in the incompressible case when the phase densities are constants). This inconsistency could have been removed simply by replacing $\alpha_{i} \rho_{i}$ with $\alpha_{i}$ in Eq. (31) (which incidentally would bring Eq. (31) into 
agreement with our Eq. (23)), but Youngs removed it in a more complicated way by introducing a distinction between mass- and volume-weighted phase velocities and postulating a further diffusional relation between the two. ${ }^{25}$

The ad hoc replacement of $\mathbf{u}_{i}$ by $\mathbf{u}_{i}-\mathbf{w}_{i}$ in Eq. (1) is unconventional and idiosyncratic, and has no apparent physical basis or justification. This modification alters the frictional forces even when the drag coefficients are not large, and, in particular, implies that those forces no longer vanish when the phase velocities $\mathbf{u}_{i}$ are equal. This behavior is at variance with the usual well-established phenomenological relations for dynamical friction. The only apparent rationale for this modification is purely mathematical: by construction, it has the desired effect of producing diffusional mass fluxes proportional to concentration gradients in the limit of large friction. As discussed above, however, the same objective could have been achieved in a much simpler, more physical, and more satisfactory manner simply by retaining the conventional form of the drag terms and including the Reynolds stress terms in their proper conservative form.

\section{The drag coefficients}

Youngs neglects the Stokes drag regime, so the drag coefficients $\beta_{i j}(u)$ are taken to be strictly linear in $u$ even for small $u$, which implies that $\beta_{i j}^{0}=0$. This may seem like a harmless simplification, but it has grave unphysical consequences when the drag coefficients are large but not infinite. The linearized Stefan-Maxwell equations (10) now become useless, so one is forced to revert to Eq. (9) instead. However, the left member of Eq. (9) is quadratic in the velocity differences, so the StefanMaxwell equations are no longer a linear system, and their solution becomes problematical except in simple special cases such as binary (two-phase) mixtures. Even worse, the resulting velocity differences are no longer linear in the driving forces $\mathbf{T}_{i}$, but rather vary like the square root of those forces. In particular, pressure diffusion fluxes are now proportional to $|\nabla p|^{1 / 2}{ }^{24,26}$ rather than $\nabla p$, which is anomalous and unphysical behavior. Similarly, concentration diffusion fluxes would have been proportional to square roots of concentration gradients if they had been allowed to emerge naturally from the isotropic parts of the Reynolds stresses as discussed above. This would have been even more obviously unphysical, but was suppressed by the assumed form of the Reynolds stress terms and the modifications to the drag forces.

\section{ACKNOWLEDGMENTS}

I am grateful to C. H. Chang for helpful discussions and thoughtful comments on the paper, and to C. H. Chang and Connie Charlton for their kind assistance in facilitating access to some of the relevant literature. I am also grateful to the anonymous referees for thoughtful and constructive comments which resulted in significant improvements.

\section{APPENDIX: REYNOLDS STRESS ANISOTROPY EFFECTS}

In Sec. III, the deviatoric Reynolds stresses $\Omega_{i}$ were omitted for simplicity in order to exhibit the origin and essence of turbulent concentration diffusion more clearly. In general, however, the effects of $\boldsymbol{\Omega}_{i}$ cannot be expected to be negligible a priori. The purpose of this Appendix is to generalize the derivation to include those effects. The generalization is straightforward and closely parallels the development in Sec. III. It is convenient to introduce the turbulent kinetic energy per unit mass tensor ${ }^{27}$ of phase $i$, which we denote by $\mathrm{K}_{i}$ and define by

$$
\mathbf{R}_{i}=-\frac{2}{3} \alpha_{i} \rho_{i} \mathbf{K}_{i},
$$

which combines with Eqs. (15) and (16) to imply $k_{i}=(1 / 3) \mathrm{K}_{i}: \mathrm{U}$, so that $k_{i} \mathrm{U}$ is the isotropic part of $\mathrm{K}_{i}$. The eigenvalues of $\mathrm{K}_{i}$ are the contributions of the individual fluctuating velocity components along its principal axes to $k_{i}$. In Sec. III, we made use of the simplifying relation $\rho_{i} k_{i}=\rho k$. The obvious generalization of this relation is $\rho_{i} \mathrm{~K}_{i}=\rho \mathrm{K} \equiv \sum_{j} \alpha_{j} \rho_{j} \mathrm{~K}_{j}$, which combines with Eq. (A1) to yield

$$
\mathrm{R}_{i}=-\frac{2}{3} \alpha_{i} \rho \mathrm{K} .
$$


The total Reynolds stress tensor of the mixture is $\mathbf{R}=\sum_{i} \mathbf{R}_{i}=-(2 / 3) \rho \mathrm{K}$, so that $\mathbf{R}_{i}=\alpha_{i} \mathbf{R}$, from which it follows that

$$
\nabla \cdot \mathrm{R}_{i}=\alpha_{i} \nabla \cdot \mathrm{R}+\mathrm{R} \cdot \nabla \alpha_{i} .
$$

The corresponding contribution to the diffusional driving forces in the Stefan-Maxwell Eqs. (10) is

$$
-\nabla \cdot \mathbf{R}_{i}+y_{i} \sum_{j} \nabla \cdot \mathbf{R}_{j}=\left(y_{i}-\alpha_{i}\right) \nabla \cdot \mathbf{R}-\mathbf{R} \cdot \nabla \alpha_{i}
$$

Comparison with the terms involving $q$ in Eq. (18) shows that restoring the previously omitted deviatoric Reynolds stresses $\boldsymbol{\Omega}_{i}$ has the effect of replacing the scalar turbulent pressure $q$ by the negative of the Reynolds stress tensor $R$. The $\nabla \cdot R$ term in Eq. (A4) represents a generalization of pressure diffusion in which the driving force is proportional to gradients of stress rather than pressure, while the $\nabla \alpha_{i}$ term represents a generalized concentration diffusion which is still driven by concentration gradients, but with tensor rather than scalar coefficients. As a result, the scalar binary diffusion coefficients $D_{i j}$ defined by Eq. (19) are replaced by tensor diffusion coefficients $D_{i j}$ of the form

$$
\mathrm{D}_{i j}=-\left(\frac{\alpha_{i} \alpha_{j}}{\beta_{i j}^{0}}\right) \mathrm{R} .
$$

Equations (19) and (A5) are turbulent analogs of the famous Einstein relation in the theory of Brownian motion ${ }^{28,29}$ which was perhaps the earliest fluctuation-dissipation theorem.

The above relations may be expressed in terms of $\boldsymbol{\Omega}_{i}$ by means of Eq. (14). When this is done, the additional terms which $\boldsymbol{\Omega}_{i}$ introduces into the right member of Eq. (18) are easily seen to be of the form

$$
\boldsymbol{\Delta}_{i} \equiv\left(y_{i}-\alpha_{i}\right) \nabla \cdot \boldsymbol{\Omega}-\boldsymbol{\Omega} \cdot \nabla \alpha_{i},
$$

while Eq. (A5) becomes

$$
\mathrm{D}_{i j}=\left(\frac{\alpha_{i} \alpha_{j}}{\beta_{i j}^{0}}\right)(q \mathrm{U}-\boldsymbol{\Omega}),
$$

where $\boldsymbol{\Omega}=\sum_{i} \boldsymbol{\Omega}_{i}$. It is of interest to consider the form these expressions assume when $\boldsymbol{\Omega}$ is modeled by means of the familiar Boussinesq eddy-viscosity hypothesis, ${ }^{12}$ according to which

$$
\boldsymbol{\Omega}=\mu_{t}\left[(\nabla \mathbf{u})+(\nabla \mathbf{u})^{\dagger}-\frac{2}{3}(\nabla \cdot \mathbf{u}) \mathrm{U}\right]
$$

Here, $\mu_{t}$ is a turbulent eddy viscosity which will ordinarily be of order $\rho \sqrt{k} \ell$, where $\ell$ is an appropriate turbulent length scale, and superscript $\dagger$ denotes the transpose. Inspection of Eqs. (A6)(A8) shows that deviatoric Reynolds stresses of Boussinesq form give rise to additional diffusional driving forces proportional to both first and second spatial derivatives of the velocity $\mathbf{u}$. The former can be described by tensor binary diffusion coefficients containing terms proportional to velocity gradients, while the latter represent turbulent shear-induced diffusion.

${ }^{1}$ J. D. Ramshaw, "Hydrodynamic theory of multicomponent diffusion and thermal diffusion in multitemperature gas mixtures," J. Non-Equilib. Thermodyn. 18, 121 (1993).

${ }^{2}$ J. D. Ramshaw and C. H. Chang, "Multicomponent diffusion in two-temperature magnetohydrodynamics," Phys. Rev. E 53, 6382 (1996).

${ }^{3}$ G. B. Wallis, One-dimensional Two-phase Flow (McGraw-Hill, New York, 1969).

${ }^{4}$ J. R. Travis, F. H. Harlow, and A. A. Amsden, "Numerical calculation of two-phase flows," Nucl. Sci. Eng. 61, 1 (1976).

${ }^{5}$ H. B. Stewart and B. Wendroff, "Two-phase flow: Models and methods," J. Comput. Phys. 56, 363 (1984).

${ }^{6}$ C. E. Brennen, Fundamentals of Multiphase Flow (Cambridge University Press, Cambridge, England, 2009).

${ }^{7}$ M. Ishii and T. Hibiki, Thermo-Fluid Dynamics of Two-Phase Flow, 2nd ed. (Springer, New York, 2011).

${ }^{8}$ J. D. Ramshaw, "Brownian motion in multiphase flow," Theor. Comput. Fluid Dyn. 14, 195 (2000).

${ }^{9}$ C. H. Chang and J. D. Ramshaw, "Pressure force transition and mixture morphology evolution in heterogeneous mixtures," Theor. Comput. Fluid Dyn. 19, 253 (2005).

${ }^{10}$ J. D. Ramshaw, "Self-consistent effective binary interaction approximation for strongly coupled multifluid dynamics," J. Non-Equilib. Thermodyn. 23, 135 (1998). 
${ }^{11}$ J. D. Ramshaw and J. A. Trapp, "Characteristics, stability, and short-wavelength phenomena in two-phase flow equation systems," Nucl. Sci. Eng. 66, 93 (1978).

${ }^{12}$ D. C. Wilcox, Turbulence Modeling for CFD, 2nd ed. (DCW Industries, La Cañada, CA, 1998)

${ }^{13}$ D. A. Drew and S. L. Passman, Theory of Multicomponent Fluids (Springer-Verlag, New York, 1999).

${ }^{14}$ D. Drew, L. Cheng, and R. T. Lahey, Jr., "The analysis of virtual mass effects in two-phase flow," Int. J. Multiphase Flow 5, 233 (1979).

${ }^{15}$ T. L. Cook and F. H. Harlow, "Virtual mass in multiphase flow," Int. J. Multiphase Flow 10, 691 (1984).

${ }^{16}$ J. D. Ramshaw, "Fluid dynamics and energetics in ideal gas mixtures," Am J. Phys. 70, 508 (2002).

${ }^{17}$ R. J. Phillips, R. C. Armstrong, R. A. Brown, A. L. Graham, and J. R. Abbott, "A constitutive equation for concentrated suspensions that accounts for shear-induced particle migration," Phys. Fluids A 4, 30 (1992).

${ }^{18}$ P. R. Nott and J. F. Brady, "Pressure-driven flow of suspensions: simulation and theory," J. Fluid Mech. 275, 157 (1994).

${ }^{19}$ J. D. Ramshaw, "Self-consistent effective binary diffusion in multicomponent gas mixtures," J. Non-Equilib. Thermodyn. 15, 295 (1990).

${ }^{20}$ J. D. Ramshaw and C. H. Chang, "Friction-weighted self-consistent effective binary diffusion approximation," J. NonEquilib. Thermodyn. 21, 223 (1996)

${ }^{21}$ E. Anderson, Z. Bai, C. Bischof, S. Blackford, J. Demmel, J. Dongarra, J. Du Croz, A. Greenbaum, S. Hammarling, A. McKenney, and D. Sorensen, LAPACK Users' Guide, 3rd ed. (SIAM, Philadelphia, 1999).

${ }^{22}$ D. C. Besnard and F. H. Harlow, "Turbulence in multiphase flow," Int. J. Multiphase Flow 14, 679 (1988).

${ }^{23}$ D. L. Youngs, "Numerical simulation of turbulent mixing by Rayleigh-Taylor instability," Physica D 12, 32 (1984).

${ }^{24}$ D. L. Youngs, "Modelling turbulent mixing by Rayleigh-Taylor instability," Physica D 37, 270 (1989).

${ }^{25}$ D. L. Youngs, "Numerical simulation of mixing by Rayleigh-Taylor and Richtmyer-Meshkov instabilities," Laser Part. Beams 12, 725 (1994).

${ }^{26}$ A. Llor, Statistical Hydrodynamic Models for Developed Mixing Instability Flows, Lecture Notes in Physics, Vol. 681 (Springer-Verlag, Berlin, 2005).

${ }^{27}$ G. K. Batchelor, The Theory of Homogeneous Turbulence (Cambridge University Press, Cambridge, England, 1970).

${ }^{28}$ R. Kubo, M. Toda, and N. Hashitsume, Statistical Physics II: Nonequilibrium Statistical Mechanics (Springer-Verlag, Berlin, 1978).

${ }^{29}$ R. Zwanzig, Nonequilibrium Statistical Mechanics (Oxford University Press, New York, 2001). 